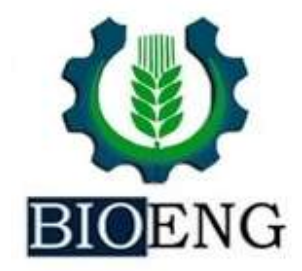

\title{
CONCENTRAÇÃO DE GLICOSE SANGUÍNEA E RELAÇÃO HETERÓFILO:LINFÓCITO PODEM SER UTILIZADOS COMO INDICADORES DE ESTRESSE TÉRMICO PARA AVES POEDEIRAS?
}

\author{
V. Kodaira ${ }^{1 *}$, D. F. Pereira ${ }^{1}$, N. M. Soares ${ }^{2}$, L. G. de F. Bueno ${ }^{3}$ \\ ${ }^{1}$ UNESP - Univ Estadual Paulista, Campus de Tupã, SP, Brasil \\ ${ }^{2}$ Instituto Biológico, Bastos, SP, Brasil \\ ${ }^{3}$ UNESP - Univ Estadual Paulista, Campus de Dracena, SP, Brasil
}

Article history: Received 19 June 2015; Received in revised form 25 June 2015; Accepted 29 June 2015; Available online 30 June 2015.

\section{RESUMO}

Aves em estresse calórico apresentam alterações fisiológicas e metabólicas para a manutenção de sua homeostase. O sistema sanguíneo é sensível a essas mudanças e a relação heterófilo:linfócito e a concentração de glicose são consideradas importantes índices de estresse crônico em aves. O objetivo da pesquisa foi verificar se a relação heterófilo:linfócito e a concentração de glicose sanguínea de aves podem ser utilizados para inferir sobre o nível de estresse das aves em diferentes condições ambientais. Foram registradas temperatura de bulbo seco, umidade relativa e temperatura de globo negro por meio de termohigrômetros, para a determinação do índice de conforto térmico de dois galpões, sendo um climatizado e outro não climatizado. As amostras de sangue de 15 aves de cada galpão foram coletadas para determinação de glicose e contagem diferencial dos leucócitos. Não houve diferença para o ITGU entre os galpões avaliados. Não se observou associações da concentração de glicose e da relação heterófilo:linfócito com o aumento do ITGU. Nas condições experimentais deste trabalho não foi possível afirmar que os parâmetros sanguíneos concentração de glicose e relação heterófilo:linfócito podem ser utilizados como indicadores de estresse térmico para aves poedeiras.

Palavras-chave: ambiência, bem-estar, parâmetros sanguíneos

\section{COULD BLOOD GLUCOSE AND HETEROPHIL:LYMPHOCYTE RATIO BE USED AS HEAT STRESS INDICATORS FOR LAYING HENS?}

\begin{abstract}
Birds in heat stress condition have physiological and metabolic changes to the maintenance of homeostasis. Blood system is sensitive to these changes and the heterophil:lymphocyte ratio and the blood glucose are considered important index for measurement of chronic stress in birds. The research aimed to verify if heterophil:lymphocyte ratio and blood glucose can be used to measure stress level in birds in different environmental conditions. Dry temperature bulb, relative humidity and black globe temperature were registered by thermo-hygrometers to determine black globe temperature and humidity index (BTHI) for two aviaries, an acclimated shed and a non-acclimated one. The blood samples of 15 birds of each shed were collected to determine the blood glucose and the heterophil:lymphocyte ratio. There was no significant difference for the BTHI between the

vkodaira@uol.com.br
\end{abstract}


sheds. There was no significant difference for blood glucose and heterophil:lymphocyte ratio with the increase of the BTHI. In this field conditions research, it was not possible to affirm that blood parameters as blood glucose and heterophil:lymphocyte ratio can be used as heat stress indicators for laying hens.

Keywords: ambience, blood parameters, welfare

\section{INTRODUÇÃO}

A exposição das aves a elevadas temperaturas dentro do aviário de postura provoca estresse por calor, que desencadeia processos fisiológicos e regulatórios na tentativa de manter ou estabelecer o estado de equilíbrio ou a homeostase (MASHALY et al, 2004; THAXTON, 2004; YAHAV et al., 2004, LIN et al., 2006, SHINI et al., 2008, STAR et al., 2008).

De acordo com BORGES (2001), o sistema sanguíneo é particularmente sensível às mudanças de temperatura e se constitui em um importante indicador das respostas fisiológicas da ave a agentes estressores. Vários indicadores são utilizados, porém a relação heterófilo:linfócito (H/L) tem sido proposta como um índice sensível de estresse crônico em aves, podendo ser esta variável mais confiável para avaliar o bem-

\section{MATERIAL E MÉTODOS}

A pesquisa foi realizada em condições de campo com poedeiras da linhagem Dekalb White alojadas a partir de 17 semanas de idade, criadas em uma granja avícola comercial de ovos localizada em Bastos, município do estado de São Paulo, Brasil, nas coordenadas $21^{\circ} 55^{\prime} 19^{\prime \prime}$ de latitude sul e $50^{\circ} 44^{\prime} 02^{\prime \prime}$ de longitude oeste, estando a uma altitude de 445 metros. De acordo com a classificação climática de Köppen, o clima é Cwa, caracterizado por clima temperado úmido com verão quente e inverno seco.

O galpão climatizado possuía 14 exaustores em uma extremidade do galpão, pad cooling na extremidade oposta $\mathrm{e}$ sistema vertical de gaiolas, com 3 baterias de 6 andares e gaiolas em ambos os lados. Em cada gaiola, cujas dimensões eram de estar de aves que a concentração de corticosterona no plasma (FURLAN \& MACARI, 2002; NICOL et al., 2009, PRIETO \& CAMPO, 2010).

Outro indicador utilizado para verificar o nível de estresse em aves é a concentração de glicose sanguínea, decorrente do aumento de glicocorticoides circulantes (BORGES et al., 2003, 2004; MUMMA et al., 2006; STAR et al., 2008, NICOL et al., 2009).

Considerando que o controle do ambiente térmico minimiza o efeito do estresse por calor, o objetivo dessa pesquisa foi verificar se a relação heterófilo:linfócito e a concentração de glicose sanguínea de aves podem ser utilizadas para inferir sobre o nível de estresse das aves nas diferentes condições ambientais.

$72 \mathrm{~cm}$ de largura e $55 \mathrm{~cm}$ de profundidade, foram dispostas 12 aves (densidade de 330 $\mathrm{cm}^{2} /$ ave/gaiola).

O galpão não climatizado possuía 2 baterias de 6 andares e gaiolas em ambos os lados. Uma bateria era composta por gaiolas de polietileno, cujas dimensões eram de $60 \mathrm{~cm}$ de largura e $53 \mathrm{~cm}$ de profundidade e foram dispostas 10 aves (densidade de $318 \mathrm{~cm}^{2} /$ ave/ gaiola). A outra bateria era composta por gaiolas de arame, de $60 \mathrm{~cm}$ de largura e $60 \mathrm{~cm}$ de profundidade, com 10 aves (densidade de $360 \mathrm{~cm}^{2} /$ ave/ gaiola).

Os valores de temperatura de bulbo seco, umidade relativa e temperatura de globo negro foram coletados por meio de 12 termohigrômetros $\mathrm{HOBO}^{\circledR}$ (Onset, Bourne, Massachusetts, EUA), instalados 
dentro dos galpões, em pontos equidistantes em todos os corredores dos galpões. $\mathrm{O}$ Índice de Temperatura de Globo Negro e Umidade (ITGU) foi calculado a partir dos dados coletados, usando a Equação 1, proposta por Buffington et al. (1981):

$$
\text { ITGU }=\mathrm{TGN}+0,36 * \mathrm{TPO}-330
$$

onde: TGN é a temperatura de globo negro (K) e TPO é a temperatura do ponto de orvalho $(\mathrm{K})$, calculado pela temperatura de bulbo seco e umidade relativa empregando equações psicrométricas.

Utilizaram-se os dados compreendidos entre o período de 01/04/2013 a 30/04/2014 e comparou-se pelo teste não paramétrico de MannWhitney a 5\% de significância.

Para a realização das análises hematológicas, foram colhidas amostras de sangue, através da punção da veia braquial de 15 aves em cada galpão a cada 28 dias, escolhidas aleatoriamente, em gaiolas distribuídas em todo o aviário, no período da manhã, para que pudessem ser processadas no mesmo dia. As análises foram realizadas no Laboratório de Patologia Avícola do Instituto Biológico de Bastos/SP. As coletas de sangue foram realizadas no período de abril de 2013 a abril de 2014.

Para dosagem de glicose plasmática foi utilizado o Kit Glicose PP (Gold Analisa, $1000 \mathrm{~mL}$ ). As amostras foram preparadas e os cálculos realizados conforme especificações do fabricante. Foi feita uma leitura para cada ave no espectrofotômetro Biochron $^{\circledR}$, modelo Libra S22 (Biochron, Cambridge, Reino Unido), com comprimento de onda de $505 \mathrm{~nm}$. Os dados foram submetidos ao teste de Mann-Whitney a 5\% de significância.

A determinação da relação heterófilo:linfócito foi realizada através da contagem de 100 leucócitos de cada esfregaço sanguíneo, diferenciando-se os linfócitos e heterófilos (Figura 1) e determinou-se a relação heterófilo:linfócito, dividindo-se o número de heterófilos encontrados pelo número de linfócitos (GONZALES et al., 2003). Os dados foram submetidos ao teste de MannWhitney a 5\% de significância.

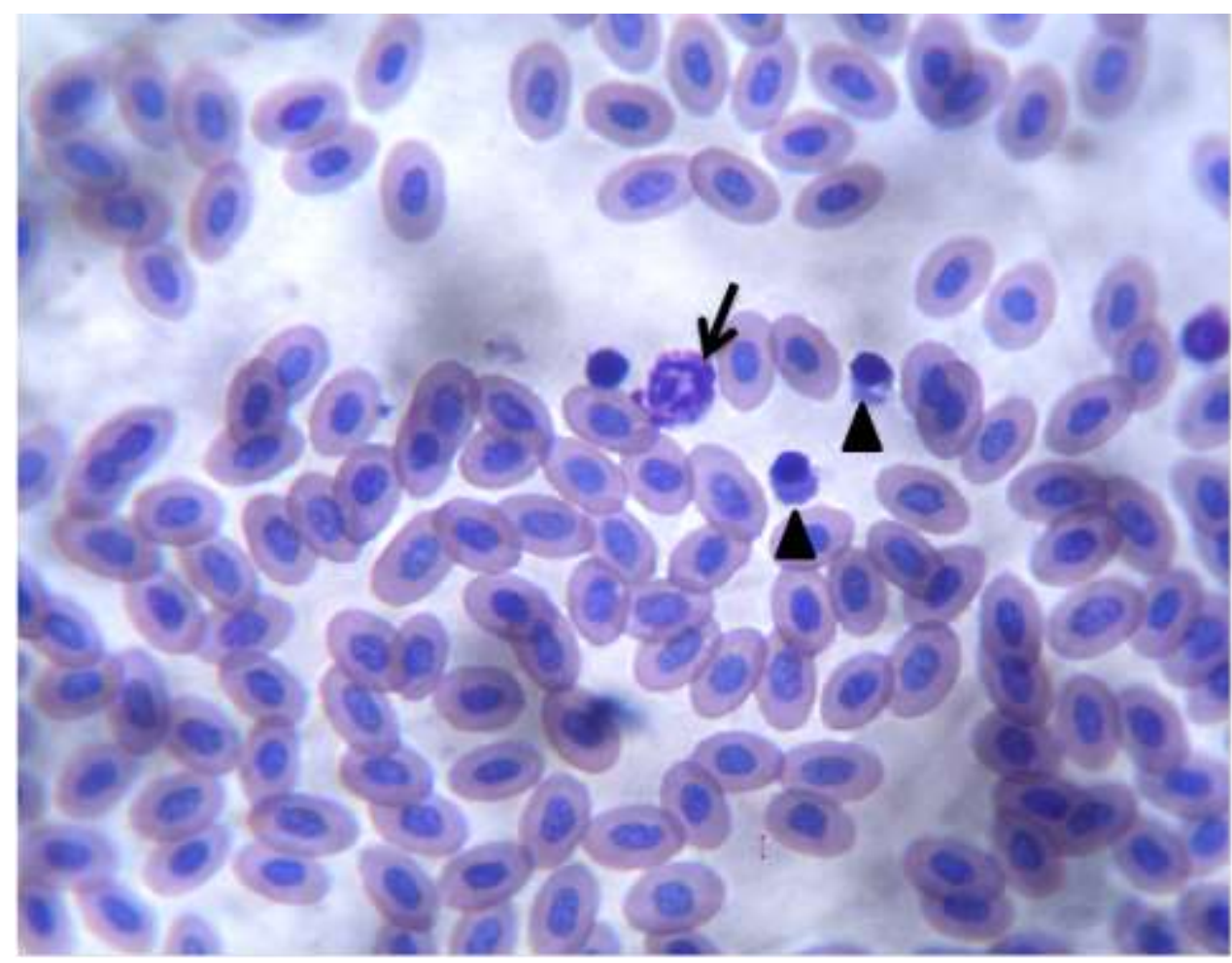

Figura 1 - Heterófilo (seta) e linfócitos (cabeça de seta). Fonte: Do próprio autor. 


\section{RESULTADOS E DISCUSSÃO}

No período total da pesquisa, verificou-se que o galpão climatizado foi eficiente na redução da temperatura e na manutenção do ITGU dentro de faixas consideradas termoneutras, quando comparado com o galpão não climatizado $(\mathrm{p}<0,05)$. Todavia, nos dias de coleta de sangue, não foram observadas diferenças $(p>0,05)$ entre os valores de ITGU de ambos os galpões no período da manhã.
Não se verificou diferença $(p>0,05)$ na concentração de glicose entre os galpões, sendo observados os valores de $198,09 \pm 44,95$ para o galpão climatizado e $198,14 \pm 53,42$ para o galpão não climatizado. Também não foi possível observar nenhuma associação do aumento de concentração de glicose com os índices de conforto térmico medidos (Figuras $2 \mathrm{e}$ $3)$.

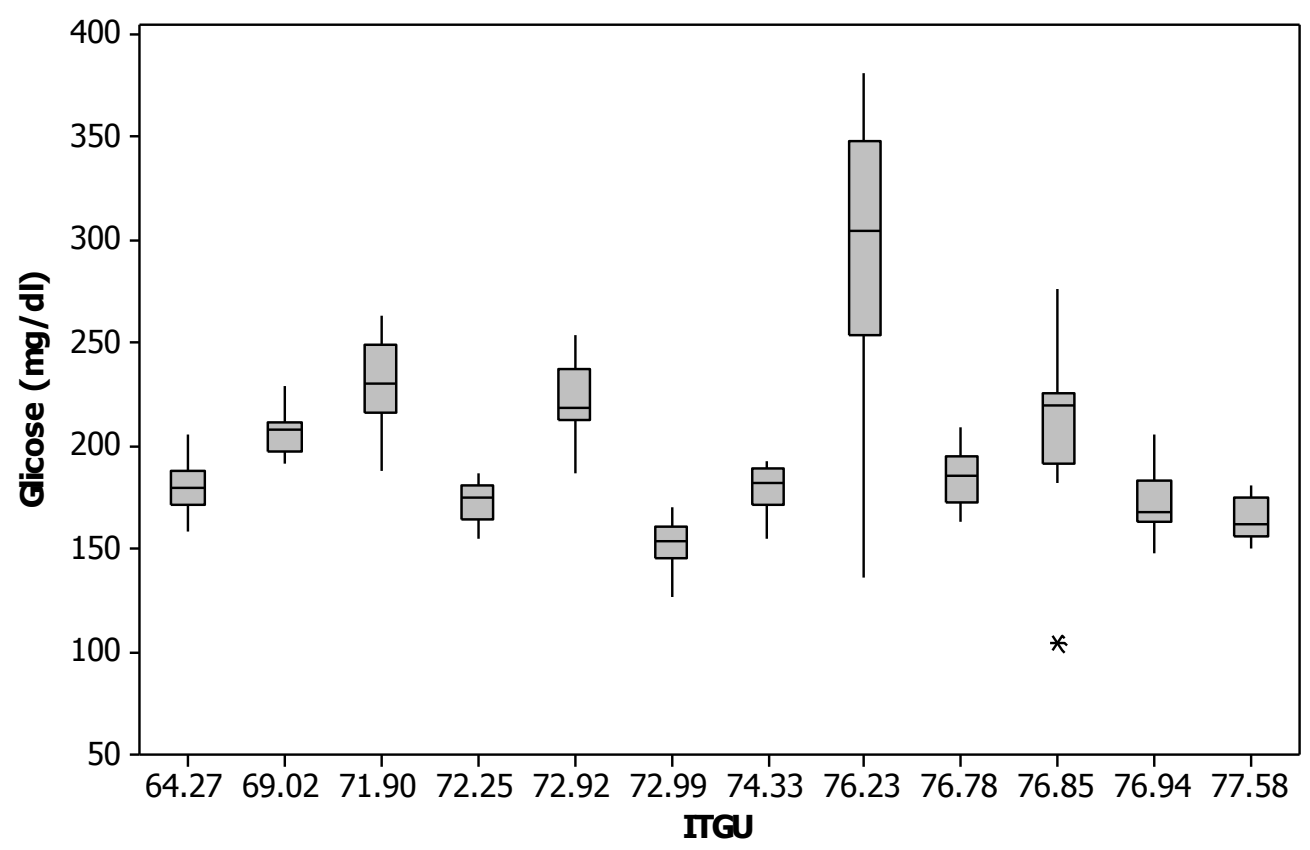

Figura 2 - Gráfico boxplot da concentração de glicose para as aves do galpão climatizado em comparação com os valores de ITGU de cada dia de coleta. 


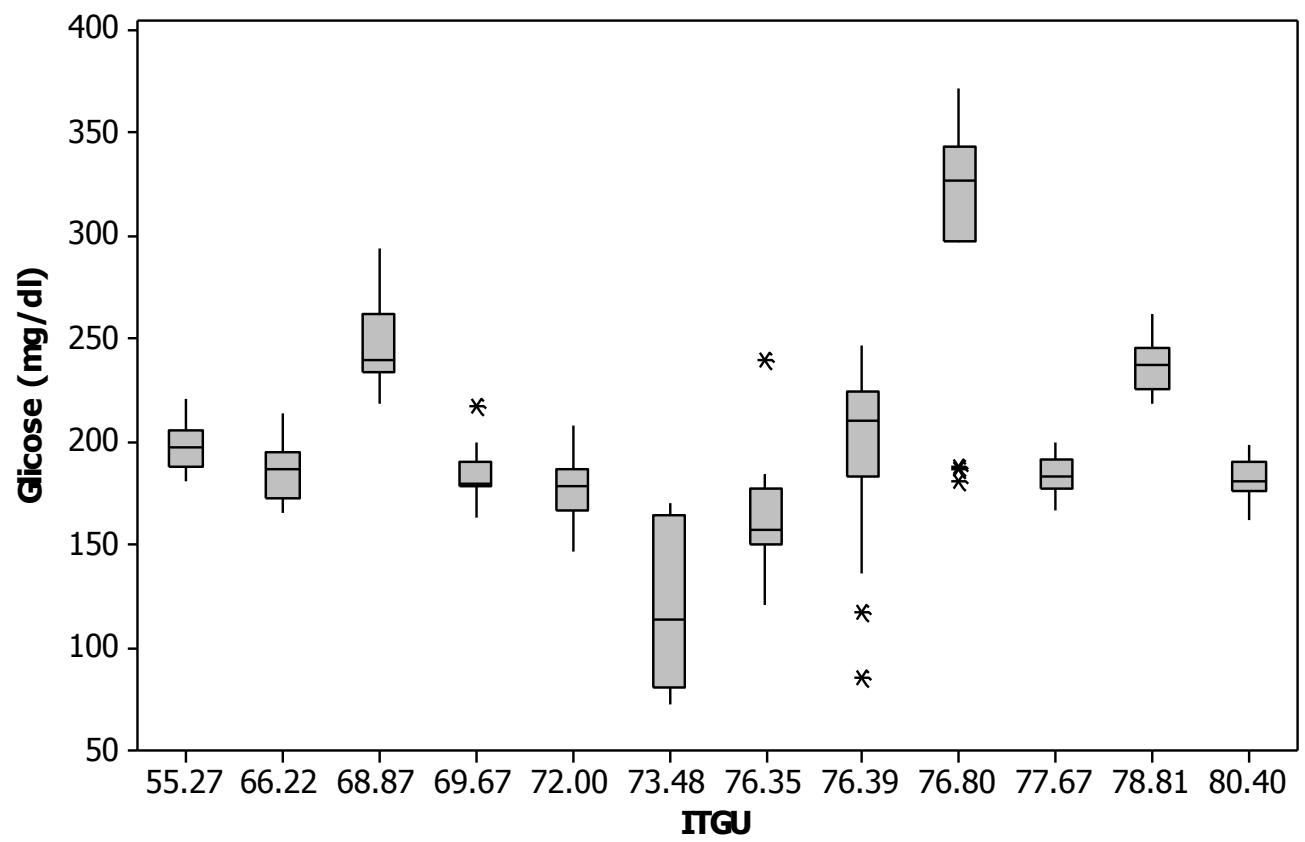

Figura 3 - Gráfico boxplot da concentração de glicose para as aves do galpão não climatizado em comparação com os valores de ITGU de cada dia de coleta.

As concentrações de glicose sanguínea de ambos os galpões apresentaram comportamento semelhante, com maior variação individual e outliers no aviário não climatizado. Não houve diferença $(p>0,05)$ entre os resultados em nenhum dos dias coletados. Além disso, as concentrações variaram entre 104 a 381 $\mathrm{mg} / \mathrm{dL}$, bem abaixo dos valores considerados em situações estressantes, que seriam acima de $594 \mathrm{mg} / \mathrm{dL}$, de acordo com Lumeji (2008).

Os resultados obtidos corroboram com BORGES et al. (2004) que, ao estudarem os efeitos do estresse calórico agudo sobre os parâmetros sanguíneos em frangos de corte, não verificaram diferenças significativas na glicose plasmática, embora tenha se aproximado da significância estatística. LIN et al. (2007) estudaram o efeito do estresse térmico em poedeiras, expondo-as à temperatura de $32^{\circ} \mathrm{C}$ durante 21 dias, sobre os níveis plasmáticos de glicose, mensurando antes da exposição, ao $1^{\circ}$ dia, 7, 14 e 21 dias após a exposição e não verificaram diferenças significativas.

Os resultados divergem dos encontrados por BORGES (2001), que ao estudar os efeitos do estresse calórico agudo sobre os parâmetros hematológicos em frangos de corte, observou aumento significativo dos níveis de glicose do grupo sob condições de estresse em relação ao grupo em condições termoneutras. GARRIGA et al. (2006) avaliaram parâmetros sanguíneos de frangos de corte mantidos por duas semanas em condições de termoneutralidade $\left(20^{\circ} \mathrm{C}\right.$ e umidade relativa $50 \%)$ e em estresse calórico $\left(30^{\circ} \mathrm{C}\right.$, umidade relativa $70 \%$ ), com ração $a d$ libitum e observaram que o segundo grupo apresentou elevação significativa de glicose plasmática. STAR et al (2008) observaram que aves submetidas a estresse calórico $\left(32^{\circ} \mathrm{C}\right)$ tiveram níveis de glicose significativamente maiores que as aves em temperatura termoneutra $\left(21^{\circ} \mathrm{C}\right) 22$ dias após o início da exposição ao calor.

Embora não esteja relacionado ao estresse térmico, mas ao estresse de ambiente físico e social, NICOL et al. (2009) alojaram 60 poedeiras sequencialmente em três ambientes (A, B e C) e compararam três conjuntos (A versus $\mathrm{B}$; $\mathrm{B}$ versus $\mathrm{C}$; A versus $\mathrm{C}$ ), durante um período de 40 semanas. Durante cada comparação, registraram mais de 100 respostas dentre comportamentais, físicas e fisiológicas, analisaram as escolhas 
ambientais entre todos os pares e observaram que os níveis de glicose foram menores quando as aves escolheram $\mathrm{o}$ melhor ambiente para elas.

$\mathrm{Na}$ relação heterófilo:linfócito não foram observadas diferenças $(p>0,05)$ entre os galpões, sendo observados os valores de $0,61 \pm 0,38$ para o galpão climatizado e $0,60 \pm 0,46$ para o galpão não climatizado (Figuras 4 e 5).

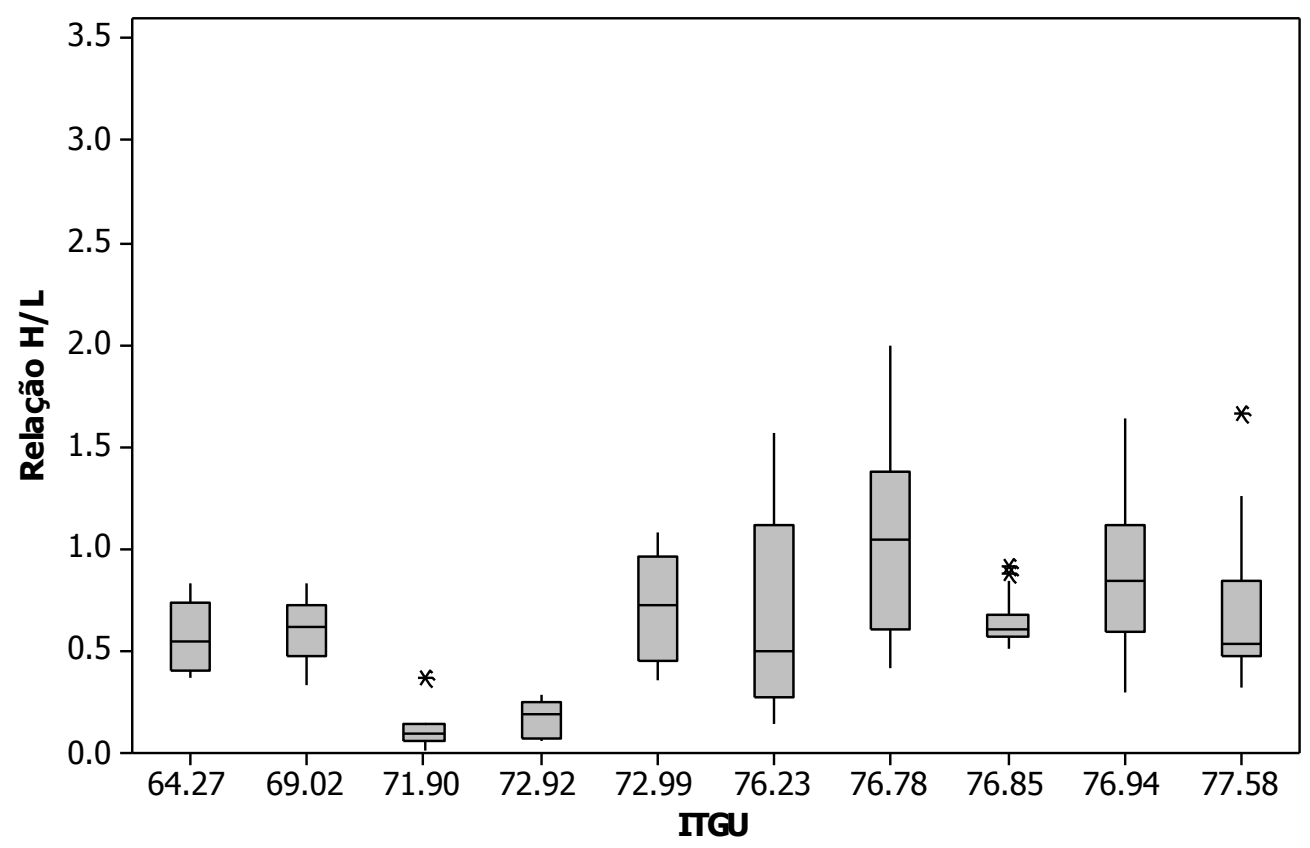

Figura 4 - Gráfico boxplot da relação heterófilo:linfócito para as aves do galpão climatizado em comparação com os valores de ITGU de cada dia de coleta.

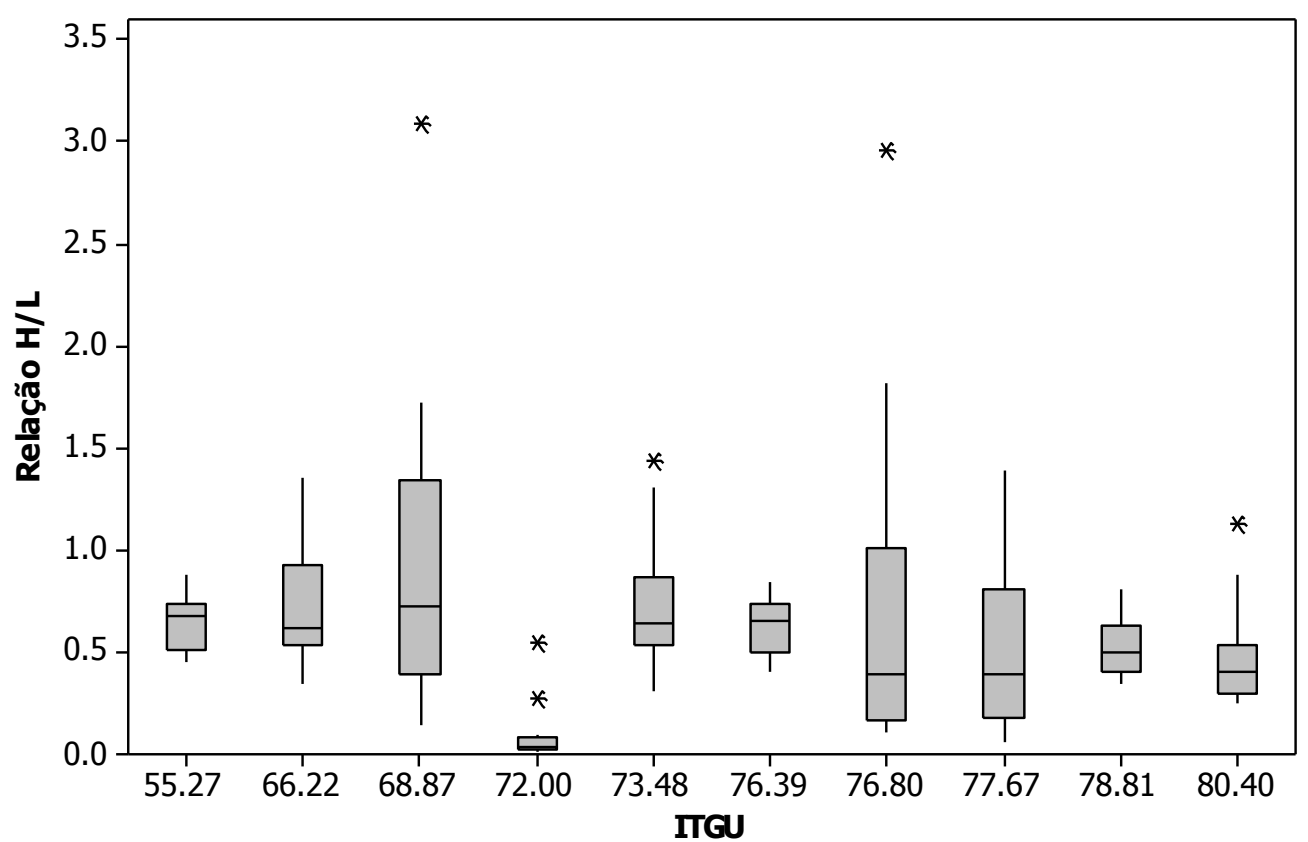

Figura 5 - Gráfico boxplot da relação heterófilo:linfócito para as aves do galpão não climatizado em comparação com os valores de ITGU de cada dia de coleta

Observa-se nas figuras 4 e 5 que não é possível estabelecer uma associação do aumento do ITGU com a relação heterófilo:linfócito $(\mathrm{H} / \mathrm{L})$. Os resultados divergem de BORGES et al. (2004), PRIETO \& CAMPO (2010) e 
SOLEIMANI et al. (2011) que observaram aumento significativo na relação $\mathrm{H} / \mathrm{L}$ de aves submetidas ao estresse calórico.

É possível que não tenha ocorrido diferença tanto para os níveis de glicose como para a relação $\mathrm{H} / \mathrm{L}$ porque as aves do galpão não climatizado já estavam aclimatadas ao calor, uma vez que a aclimatação ocorre em um período de 4 a 7 dias de exposição a altas temperaturas (YAHAV, 2009). LAGANÁ et al. (2007) verificaram a concentração de glicose e a relação H/L, em frangos de corte submetidos ao estresse por calor e alimentação ad libitum de 21 a 42 dias de idade. Não houve aumento da glicose com o estresse térmico em relação ao grupo controle e a relação $\mathrm{H} / \mathrm{L}$ esteve próxima aos valores considerados normais de 0,5 (MACARI \& LUQUETI, 2002), concluindo o efeito da aclimatação sobre as aves influenciou os resultados.

A coleta de sangue foi realizada no período da manhã e não se observou diferenças entre os valores de ITGU. Isso pode ter influenciado no resultado obtido para concentração de glicose sanguínea, uma vez que ela é utilizada como indicador

\section{CONCLUSÕES}

Não se verificou associação entre a concentração de glicose e o ambiente térmico e entre a relação heterófilo:linfócito e o ambiente térmico.

\section{AGRADECIMENTOS}

Os autores agradecem à FAPESP Processo No. 2013/09706-3 pelo auxílio à pesquisa, à Granja Yoshikawa por

\section{REFERÊNCIAS}

BORGES, S. A. Balanço eletrolítico e sua interrelação com o equilíbrio ácido-base em frangos de corte submetidos a estresse calórico. 2001. 97 f. Tese (Doutorado em Zootecnia) - Faculdade de Ciências Agrárias e Veterinária, em caso de estresse agudo ou crônico. Não houve coletas de sangue em dias que houve queda do fornecimento de energia elétrica, nem no período da tarde, quando se verificou diferenças significativas nos valores de ITGU entre os galpões.

$\mathrm{O}$ estresse social e hierárquico entre as aves pode afetar a relação $\mathrm{H} / \mathrm{L}$, pois tanto no aviário climatizado como no não climatizado, elas estavam alojadas em grupos de 12 e 10 aves por gaiola, respectivamente. MOE et al. (2010), verificaram que grupos de 8 aves apresentaram aumento significativo da relação $\mathrm{H} / \mathrm{L}$ quando comparados com grupos de 3 aves por gaiola.

Fatores como idade, agentes infecciosos, presença de pessoas, relação entre aves e humanos nos galpões (GROSS \& SIEGEL, 1983) e a variabilidade individual (SANTOS et al., 2013), também interferem na relação H/L. Uma infestação maciça por ectoparasitas nas aves presente durante quase todo o período da pesquisa e a coleta realizada por pessoas estranhas ao convívio das aves podem ter influenciado nos resultados.

Nas condições experimentais deste trabalho não foi possível afirmar que os parâmetros estudados podem ser utilizados como indicadores de estresse térmico para aves poedeiras.

permitirem a realização do experimento em suas instalações e ao Instituto Biológico de Bastos pela realização das análises.

Universidade Estadual Paulista, Jaboticabal, 2001.

BORGES, S. A.; MAIORKA, A.; SILVA, A. V. F. Fisiologia do estresse calórico e a utilização de eletrólitos em frangos de 
corte. Ciência Rural, v. 33(5): 975-981, set./out., 2003.

BORGES, S. A.; SILVA, A. V. F.; MAIORKA, A.; HOOGE, D. M.; CUMMINGS, K. R. Physiological responses of broiler chickens to heat stress and dietary electrolyte balance (sodium plus potassium minus chloride, milliequivalents per kilogram). Poultry Science, v. 83: 1551-1558, 2004.

BUFFINGTON, C. S.; COLLIER, R. I.; CANTON, G. H. Shade management system heat stress for dairy cows in hot, humid climates. Transactions of the ASAE, v. 26(6): 1798-1802, 1981.

FURLAN, R. L.; MACARI, M. Termorregulação. In: MACARI, M.; FURLAN， R. L.; GONZALES, E. Fisiologia aviária aplicada a frangos de corte. Jaboticabal: FUNEP/UNESP, 2002. p. 209-230.

GARRIGA, C.; HUNTER, R. R.; AMAT, C.; PLANAS, J. M.; MITCHELL, M. A.; MORETÓ, M. Heat stress increases apical glucose transport in the chicken jejunum. American Journal of Physiology Regulatory, Integrative and Comparative Physiology, v. 290: R195-R201, 2006.

GONZALES, E. et al. Performance and physiological parameters of broiler chickens subjected to fasting on the neonatal period. Poultry Science, 82: 1250-1256, 2003.

GROSS, W. B.; SIEGEL, H. S. Evaluation of heterophil/lymphocyte ratio as a measure of stress in chickens. Avian Diseases, v. 27(4): 972-979, oct./dec., 1983.

LAGANÁ, C.; RIBEIRO, A. M., L.; GONZÁLEZ, F. H. D.; LACERDA, L. A.; KRATZ, L. R.; BARBOSA, P. R. Níveis dietéticos de proteína e gordura e parâmetros bioquímicos, hematológicos e empenamento em frangos de corte estressados pelo calor. Revista Brasileira de Zootecnia, v. 36(6): 1783-1790, 2007.

LIN, H.; JIAO, H. C.; BUYSE, J.; DECUYPERE, E. Strategies for preventing heat stress in poultry. World's Poultry Science Journal, v. 62: 71-85, mar. 2006.

LIN, H.; DE VOS, D.; DECUYPERE, E.; BUYSE, J. Dynamic changes in parameters of redox balance after mild heat stress in aged laying hens (Gallus gallus domesticus). Comparative Biochemistry and Physiology, Cambridge, Part C 147: 30-35, 2007.

LUMEJI, J. T. Avian clinical biochemistry. In: KANEKO, J. J.; HARVEY, J. W.; BRUSS, M. L. Clinical Biochemistry of Domestic Animals. Oxford: Elsevier Academic Press, 2008. p. 839-872.

MACARI, M.; LUQUETTI, B. C. Fisiologia cardiovascular. In: MACARI, M.; FURLAN, R. L.; GONZALES, E. Fisiologia aviária aplicada a frangos de corte. Jaboticabal: FUNEP/UNESP, 2002. p. 17-35.

MASHALY, M. M.; HENDRICKS, G. L.; KALAMA, M. A.; GEHAD, A. E.; ABBAS, A. O.; PATTERSON, P. H. Effect of heat stress on production parameters and immune responses of commercial laying hens. Poultry Science, v. 83: 889-894, 2004.

MUMMA, J. O.; THAXTON, J. P.; VIZZIER-THAXON, Y.; DODSON, W. L. Physiological stress in laying hens. Poultry Science, v. 85: 761-769, 2006.

MOE, R. O.; GUÉMENÉ, D.; BAKKEN, M.; LARSEN, H. J. S.; SHINI, S.; LERVIK, S.; SKJERVE, E.; MICHEL, V.; TAUSON, R. Effects of housing conditions during the rearing and laying period on adrenal reactivity, immune response and heterophil to lymphocyte $(\mathrm{H} / \mathrm{L})$ ratios in laying hens. Animal, v. 4(10): 1709-1715, 2010.

NICOL, C. J.; CAPLEN, G.; EDGAR, J.; BROWNE, W. J. Associations between welfare indicators and environmental choice in laying hens. Animal Behaviour, v. 78: 413-424, 2009.

PRIETO, M. T.; CAMPO, J. L. Effect of heat and several additives related to stress levels on fluctuating asymmetry, 
heterophil:lymphocyte ratio, and tonic immobility duration in White Leghorn chicks. Poultry Science, v. 89: 2071-2077, 2010.

SANTOS, S. C. S; MEYER, R.; COSTA, M. F. D. Variety of haematological parameters of monthly blood donor dogs.

Revista de Ciências Médicas e Biológicas,

v.12: 472-477, dez.2013.

SHINI, S.; KAISE, P.; SHINI, A.; BRYDEN, W. L. Differential alterations in ultrastructural morphology of chicken heterophils and lymphocytes induced by corticosterone and lipopolysaccharide. Veterinary Immunology and Immunopathology, v. 122: 83-93, 2008.

SOLEIMANI, A. F.; ZULKIFLI, I.;OMAR, A. R.; RAHA, A. R. Physiological responses of 3 chicken breeds to acute heat stress. Poultry Science, v. 90: 1435-1440, 2011.

STAR, L.; DECUYPERE, E.; PARMENTIER, H. K.; KEMP, B. Effect of single combined climatic and hygienic stress in four layer lines: 2. Endocrine and oxidative stress responses. Poultry Science, v. 87: 1031-1038, 2008.

THAXTON, J. P. Welfare of the laying hen. Poultry Science, v. 27: 81-95, 2004.

YAHAV, S.; STRASCHNOW, A.; LUGER, D.; SHINDER, D.; TANNY, J; COHEN, S. Ventilation, sensible heat loss, broiler energy, and water balance under harsh environmental conditions. Poultry Science, v. 83: 253-258, 2004.

YAHAV, S. Alleviating heat stress in domestic fowl: different strategies. World's Poultry Science Journal, v. 65: 719-732, dec. 2009. 